\title{
INEQUALITIES WITH CURVATURE AND THEIR STABILITY ESTIMATES FOR CONVEX CURVES
}

\section{DEYAN ZHANG}

Abstract. In this paper, we deal with a geometric inequality for a closed convex plane curve $\gamma$ involving the area, the perimeter, the curvature of $\gamma$. Together with an inequality obtained by Lin and Tsai in [12], we conclude the upper and lower bound estimates for the integration of the squared curvature radius of $\gamma$ and show the stability results of the corresponding inequalities. Mathematics subject classification (2010): 52A38, 52A40.

Keywords and phrases: Stability, convex plane curve, geometric inequality, Fourier series.

\section{REFERENCES}

[1] V. I. Diskant, Stability of the solution of the Minkowski equation, Siberian Math. J., 14 (1973), 466-469.

[2] B. Fuglede, Stability in the isoperimetric problem, Bull. London Math. Soc., 18 (1986), 599-605.

[3] M. GAGE, An isoperimetric inequality with applications to curve shortening, Duke. Math. J., 50, 4 (1983), 1225-1229.

[4] M. GAGE, Curve shortening makes convex curves circular, Invent. Math., 76, 2 (1984), 357-364.

[5] M. Gage, On an area-preserving evolution equation for plane curves, Contemp. Math., 51 (1986), $51-62$.

[6] M. Gage And R. Hamilton, The heat equation shrinking convex plane curves, J. Diff. Geom., 23, 1 (1986), 69-96.

[7] X. GAO, A new reverse isoperimetric inequality and its stability, Math. Inequalities and Appl., 12, 3 (2012), 733-743.

[8] H. Groemer, Stability theorems for convex domains of constant width, Canad. Math. Bull., 31 (1988), 328-337.

[9] H. Groemer AND R. SCHNEIDER, Stability estimates for some geometric inequalities, Bull. London Math. Soc., 23 (1991), 67-74.

[10] H. Groemer, Stability theorems for projections of convex sets, Israel J. Math., 60, 2 (1987), 177-190.

[11] Q. Guo, Stability of the Minkowski measure of asymmetry for convex bodies, Discrete Comput. Geom., 34 (2005), 351-362.

[12] Y. C. Lin AND D. H. Ts AI, Application of Andrews and Green-Osher inequalities to nonlocal flow of convex plane curve, J. Evol. Equ., 12 (2012), 833-854.

[13] S. L. PAn And H. P. XU, Stability of a reverse isoperimetric inequality, J. Math. Anal. Appl., 350 (2009), 348-353.

[14] S. L. PAN AND J. N. YANG, On a non-local perimeter-preserving curve evolution problem for convex plane curves, Manuscripta Math., 127 (2008), 469-484.

[15] R. SCHNEIDER, On Steiner points of convex bodies, Israel J. Math., 9 (1971), 241-249.

[16] R. SCHNEIDER, Stability in the Aleksandrov-Fenchel-Jesson theorem, Mathematika, 36 (1989), 5059.

[17] R. SCHNEIDER, A stability estimate for the Aleksandrov-Fenchel inequality, with an application to mean curvature, Manuscripta Math., 69 (1990), 291-300.

[18] J. STEINER, Sur le maximum et le minimum des figures dans le plan, sur la sphère, et dans l'espace en général, I and II, J. Reine Angew. Math. (Crelle), 24 (1842), 93-152 and 189-250. 